Provided for non-commercial research and education use. Not for reproduction, distribution or commercial use.

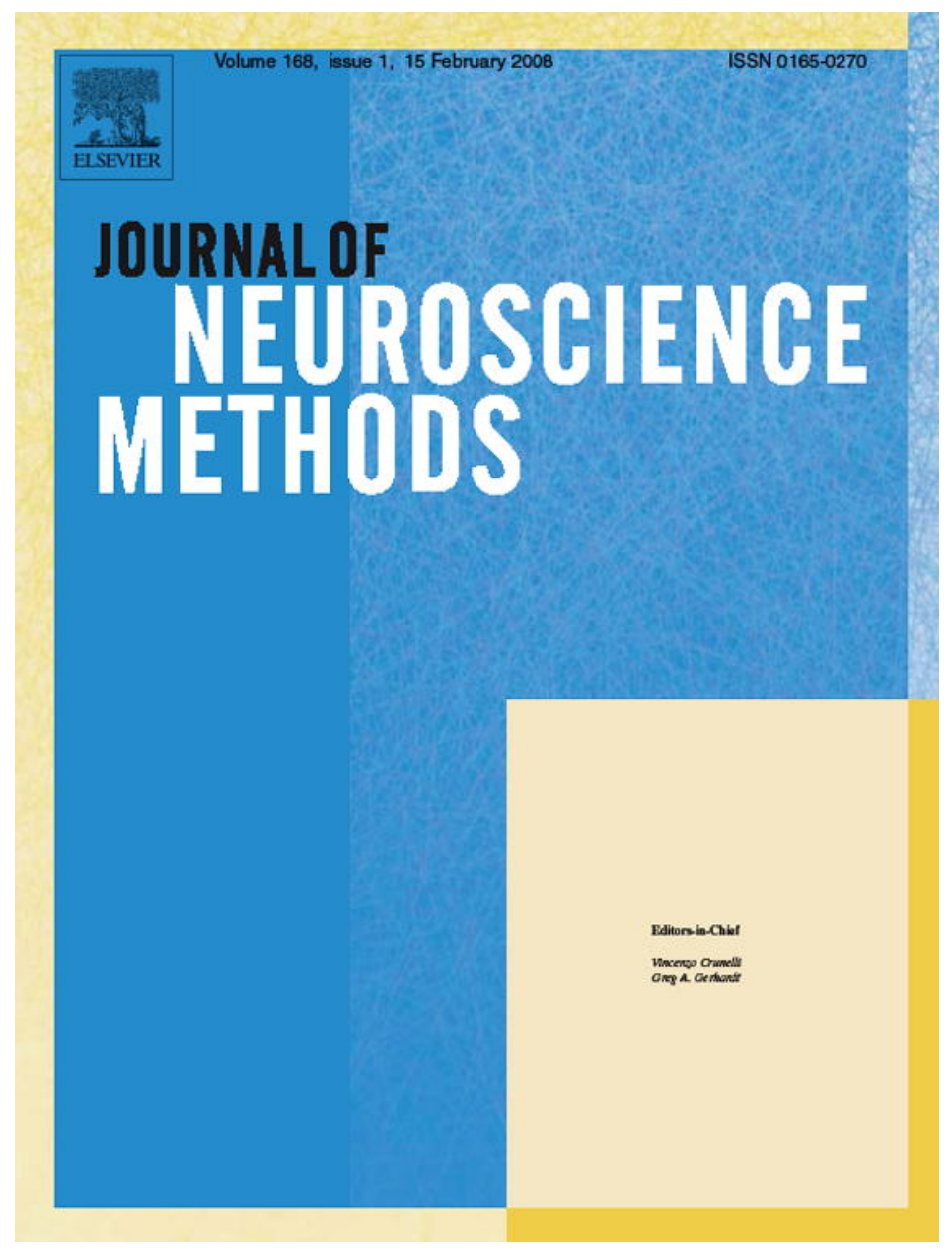

This article was published in an Elsevier journal. The attached copy

is furnished to the author for non-commercial research and education use, including for instruction at the author's institution, sharing with colleagues and providing to institution administration.

Other uses, including reproduction and distribution, or selling or licensing copies, or posting to personal, institutional or third party websites are prohibited.

In most cases authors are permitted to post their version of the article (e.g. in Word or Tex form) to their personal website or institutional repository. Authors requiring further information regarding Elsevier's archiving and manuscript policies are encouraged to visit:

http://www.elsevier.com/copyright 


\title{
A dichoptic projection system for visual psychophysics in fMRI scanners
}

\author{
Benjamin Thompson $^{\mathrm{a}, *}$, Reza Farivar ${ }^{\mathrm{a}, \mathrm{b}}$, Bruce C. Hansen ${ }^{\mathrm{a}, 1}$, Robert F. Hess ${ }^{\mathrm{a}}$ \\ ${ }^{a}$ McGill Vision Research, Department of Ophthalmology, McGill University, 687 Pine Avenue West, Montreal, Quebec, Cananda H3A 1A1 \\ ${ }^{\mathrm{b}}$ Psychology Department, McGill University, Canada
}

Received 25 July 2007; received in revised form 17 September 2007; accepted 17 September 2007

\begin{abstract}
Here we describe a projection system based on the combination of linear polarizing filters, designed to allow dichoptic presentation of visual stimuli during fMRI experiments. Currently available MRI compatible dichoptic presentation systems are either highly expensive, require degradation of the projected stimulus such as the removal of all but one color or are difficult to deploy in a range of scanner environments. The system described here is relatively low in cost, requires no change in stimulus properties and could be used in many types of scanner facilities. We provide a verification of the system demonstrating minimal cross-talk between the images presented to the two eyes.
\end{abstract}

(c) 2007 Elsevier B.V. All rights reserved.

Keywords: Dichoptic; Binocular; Stimulus presentation; fMRI; Projection system; Duel projection; Polarization

\section{Introduction}

The way in which the human visual system behaves when stimuli are presented independently to each eye (dichoptic presentation) is of interest to researchers investigating the mechanisms underlying visual perception (Baker et al., 2007; Harrad and Hess, 1992; Levi et al., 1979; Li et al., 2005; Macknik and Martinez-Conde, 2004; Meese et al., 2006; Sengpiel, 1997; Sengpiel and Vorobyov, 2005). Functional magnetic resonance imaging (fMRI) can be combined with dichoptic stimulation procedures to explore the neural responses of the human brain under different perceptual states (Tong et al., 2006). A critical component of any fMRI experiment concerned with visual psychophysics is the accurate presentation of the stimuli to be tested. In the laboratory there are multiple ways in which stimuli can be presented dichoptically without having to alter the properties of either image. Such techniques include shutter goggles, polarized screen overlays and stereoscopes. However, none of these techniques are easily implemented within the MR environment because of either their size, the requirements of a CRT monitor overlay or the presence of numerous ferromagnetic components.

\footnotetext{
* Corresponding author. Tel.: +1 514 9341934x35307; fax: +1 5148431691. E-mail address: ben.thompson@mcgill.ca (B. Thompson).

${ }^{1}$ Present address: Department of Psychology, Colgate University, Hamilton, NY 13346, USA.
}

Researchers have two primary choices when considering dichoptic fMRI stimulus presentation techniques. The first is to use a projection system whereby the subject views a projected image, positioned at either the head or the foot of the scanner bore, through a mirror or a prism system mounted just above the eyes. The second option is to use a commercially available goggle system exploiting LCD or optic fiber technology to present the stimulus on small screens in front of each eye. Each system has its own benefits and drawbacks. Projection systems allow for much finer control over the properties of the stimulus being presented, such as luminance (which can be directly measured with a photometer), visual angle and viewing distance and are therefore often the preferred choice for experiments requiring accurate psychophysical stimulation. In addition, projection systems can be removed from the scanner room and used for training or measurement purposes without requiring access to the scanning area. Another consideration is the fact that projection systems are substantially less expensive than fMRI compatible goggles, which require specialized nonferromagnetic components and have to be custom installed into the scanner room. One drawback with using projection systems is that it is not straightforward to present stimuli separately to each eye without sacrificing certain properties of the images, a problem easily overcome with fMRI compatible goggles. Studies that have used projection systems for dichoptic stimulation have typically used anaglyph spectacles in order to present separate images to each eye (Backus et al., 2001; Brouwer et al., 
2005; Chandrasekaran et al., 2007; Fang and He, 2005; Haynes et al., 2005; Haynes and Rees, 2005; Meng et al., 2005; Moutoussis et al., 2005; Polonsky et al., 2000; Tong et al., 1998; Wunderlich et al., 2005). This technique, which uses different colored gels over each eye to filter out one of the two colors presented in the projected image, works well in many situations, however, it does require that the stimuli are presented in a degraded state and it is often challenging to fully remove "cross-talk" between each eye. Therefore, if characteristics such as color are important to the experimental procedure, this way of presenting the stimuli is not always appropriate. An alternative technique is to view the stimuli though a pair of fMRI compatible binoculars equipped with individual mirrors on the end of each lens which can be adjusted to view only one half of the presentation screen (Brouwer et al., 2005; Polonsky et al., 2000). In this way, the generation of two spatially separated images on the presentation screen can allow for two separate monocular images to be presented to each eye provided that a septum is held between the knees to prevent one eye seeing a portion of the other eye's image. This technique is effective, but can be difficult to implement for different types of head coils and for rear projection systems. In addition, using only half the potential presentation area effectively halves the spatial resolution at which the image could be projected. We therefore developed an alternative, low cost, fMRI compatible dichoptic presentation technique using two projectors and a set of polarizing filters to simultaneously project full-field images separately to each eye. We have successfully used this system to evaluate binocular suppression in Amblyopia using a Siemens Sonata $1.5 \mathrm{~T}$ scanner with a posterior flex coil (Thompson et al., 2007). This imaging system had a full-body bore with a diameter of $61.5 \mathrm{~cm}$, which allowed for a field of view of $34^{\circ}$ of visual angle.

\section{Materials and methods}

\subsection{Projector specifications and mounting}

Two identical InFocus LP460 projectors (InFocus Corporation, OR, USA) were mounted on a custom stand (Fig. 1) that allowed for the images projected by the two machines to be precisely overlaid on one another. The stand consisted of two platforms, both of which allowed adjustments in two-dimensions (pitch and roll). The lower platform also had a yaw control to provide additional alignment adjustment and also to allow for a disparity to be introduced between the two images if required. All components used in the stands were non-ferromagnetic to allow for MRI compatibility. Keystoning functions already built into the projectors were employed to cancel out the differences in image aspect ratio resulting from the slightly different vertical angles at which the two projectors were oriented.

\subsection{Polarization}

Linear polarizing filters were mounted in custom casings in front of each projector's lens. The polarizers were $50 \mathrm{~mm}$ in diameter and $2 \mathrm{~mm}$ thick. According to the distributor's (Edmund Optics, Barrington, USA) specifications, each filter

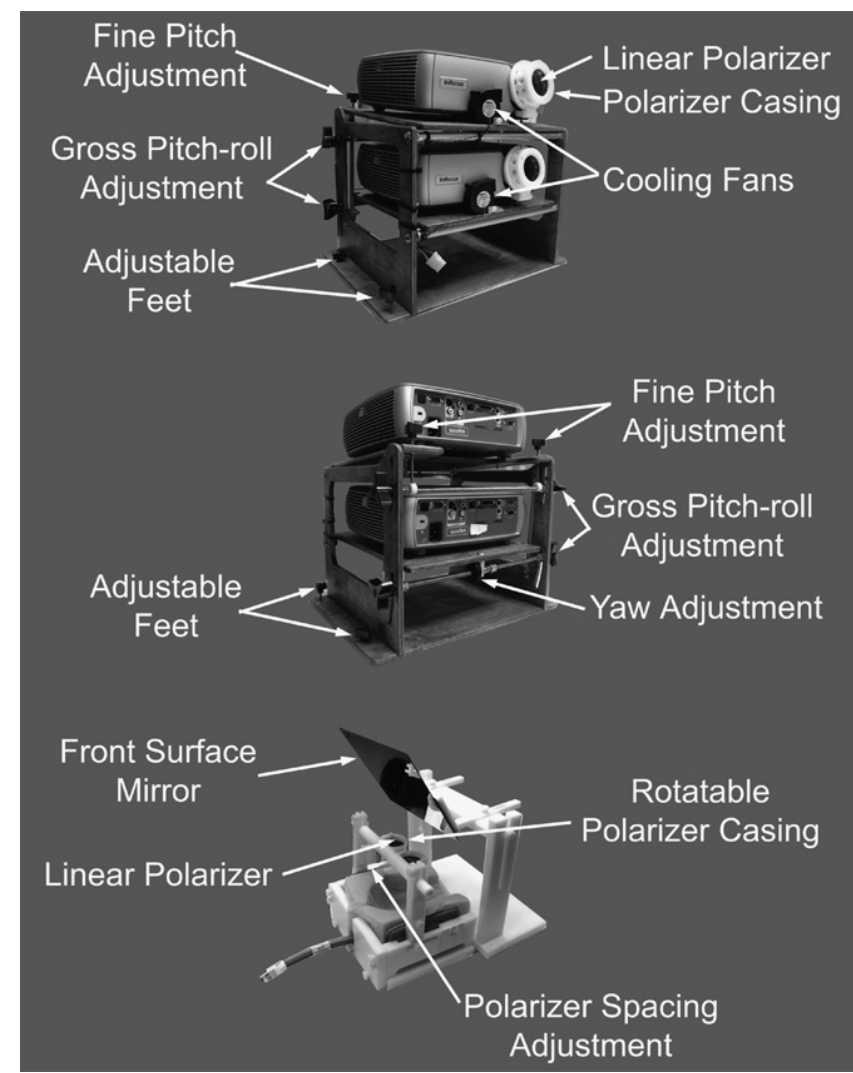

Fig. 1. Front and rear views of the projection assembly and a view of the mirror and polarizer holders mounted for use with a surface coil.

had an average transmission of $25 \%$ within the $400-700 \mathrm{~nm}$ range. Pairs of polarizers had an extinction ratio of 10,000:1 and the axis of polarization was marked on the edge of each polarizer by the manufacturer which facilitated alignment for optimum extinction. The casings allowed the polarizers to be rotated so that the polarization angle could be adjusted. In addition, each casing tilted to allow the polarizer to be aligned with the center of the projected beam. This was necessary as the projectors did not project through the very center of the lens, but at an upward angle to facilitate projection from table-top or ceiling mounted positions. Therefore, a tilt in the polarizer was required to ensure that the projected light passed through it at a perpendicular angle and also to minimize the effect of the polarizer mounting on the size of the projected image. When correctly positioned, the polarizer casings removed only the outer corners of the projected images. The polarizer casings also contained a panel of heat absorbing glass since the heat generated close to the projector was sufficient to burn a hole in the polarized coating of the lenses. Holes were also drilled in the mounting to enable airflow and small fans were mounted at the side of each casing to provide a flow of cooler air. This allowed the projection system to run indefinitely without the projectors or lenses overheating. The heat absorbing glass was $51 \mathrm{~mm}$ in diameter and $6.4 \mathrm{~mm}$ thick and absorbed wavelengths beyond the visible spectrum, dissipating the heat into the surrounding air. According to distributor's (Edmund Optics, Barrington, USA) specifications, the Schott KG-1 glass disk had a flat transmission distribution 
of $85 \%$ over the $350-650 \mathrm{~nm}$ range and therefore had little influence on spectral properties of the light projected through it by the LCD projectors since LCD projectors emit little energy in the $650-700 \mathrm{~nm}$ range.

Since LCD projectors pre-polarize the light that they project, with red and blue light projected with a vertical polarity and green light projected with a horizontal polarity (Kim and Kim, 2006), it was important to rotate the polarizers mounted on the projectors so that the orientation of polarization was at precisely $\pm 45^{\circ}$ from vertical to maintain full color balance for both images. The LP640 projector has markings at both of these orientations that facilitate filter alignment. As this alignment attenuated light equally over all three wavelengths, the luminance of the image was reduced. This proved not to be a problem however as the projected light had a sufficiently high initial luminance that a reduction did not adversely affect the projected images and in fact made viewing more comfortable from within the scanner bore.

Both polarized images were back projected onto a rear projection screen (Eclipse II, Reversa World SL, Buganvillas, Spain) specifically designed to maintain the polarization of the light projected through it. As these screens are typically designed to maximally preserve polarization in vertical and horizontal planes we found it necessary to rotate the screen by approximately $45^{\circ}$ to optimize the polarization of our images. The screen was held in place directly abutting the scanner bore using Velcro strips.

\subsection{Viewing}

The rear-projected images were viewed by the observer through linear polarizers identical to those mounted over the projector lenses. The polarizers were mounted in a plastic, MRI compatible frame which allowed each polarizer to be rotated independently to extinguish the image projected to the other eye. The frame also allowed for the lens pitch, roll and angle to be adjusted. In addition, the spacing between the lenses could also be adjusted to make the subject as comfortable as possible. From within the bore of the MRI scanner, the projection screen could not be viewed directly and therefore had to be viewed via a mirror. Standard back surface glass mirrors were found not to hold the polarization of the projected light sufficiently to allow for complete extinction of the two images. Therefore, a front surface mirror was used to maintain polarity. According to distributor's (Edmund Optics, Barrington, USA) specifications, the mirror was coated with enhanced aluminum with a $4-6 \lambda$ per inch surface accuracy, providing $>90 \%$ reflection between 400 and $650 \mathrm{~nm}$. Mirror and polarizer assemblies were attached to a custom head coil mount which held a surface coil. In principle, nothing prevents the adaptation of this system for use with other types of head coils including full head coils. Naturally, design considerations would need to be made such as the size of the head-mounted polarizers and the associated rotatable casings, as well as the exact shape and size of the mirror. For a full-head coil, the mirror itself would have to be greatly reduced in size. If a standard mirror is currently used in conjunction with a projection system within an imaging facility using such a coil, this could be used as a template for the specialized mirror required for the current system. The polarizers above the eyes could be placed either inside the coil, as is often the case with goggle systems, or above the coil depending on the position of the mirror and the amount of space available between the head coil and the top of the scanner bore. We found that the magnetic field generated by the scanner did not affect the performance of the polarization systems. We also found, using a phantom, that the presence of the front surface mirror in close proximity to the surface coil did not cause any artifacts in functional or structural image acquisition protocols.

\subsection{Projection system output verification}

The projection system was set up in a darkened laboratory. Both projectors were gamma corrected using an OptiCAL photometer (Cambridge Research Systems, Rochester, UK) fitted with a $1 \log$ unit neutral density filter (1 N.D. Wratten gelatin filter, Kodak, New York) to prevent the luminance exceeding the range within which the photometer could make accurate measurements. Luminance readings were taken at 18 gray levels in four different conditions. Firstly, luminance was measured on the viewing side of the rear projection screen with one projector projecting through a single $45^{\circ}$-orientated polarizer located directly in front of the projector itself (Fig. 2A). A set of measurements were then made with the addition of a second polarizer which was open to assess the reduction of luminance reach-

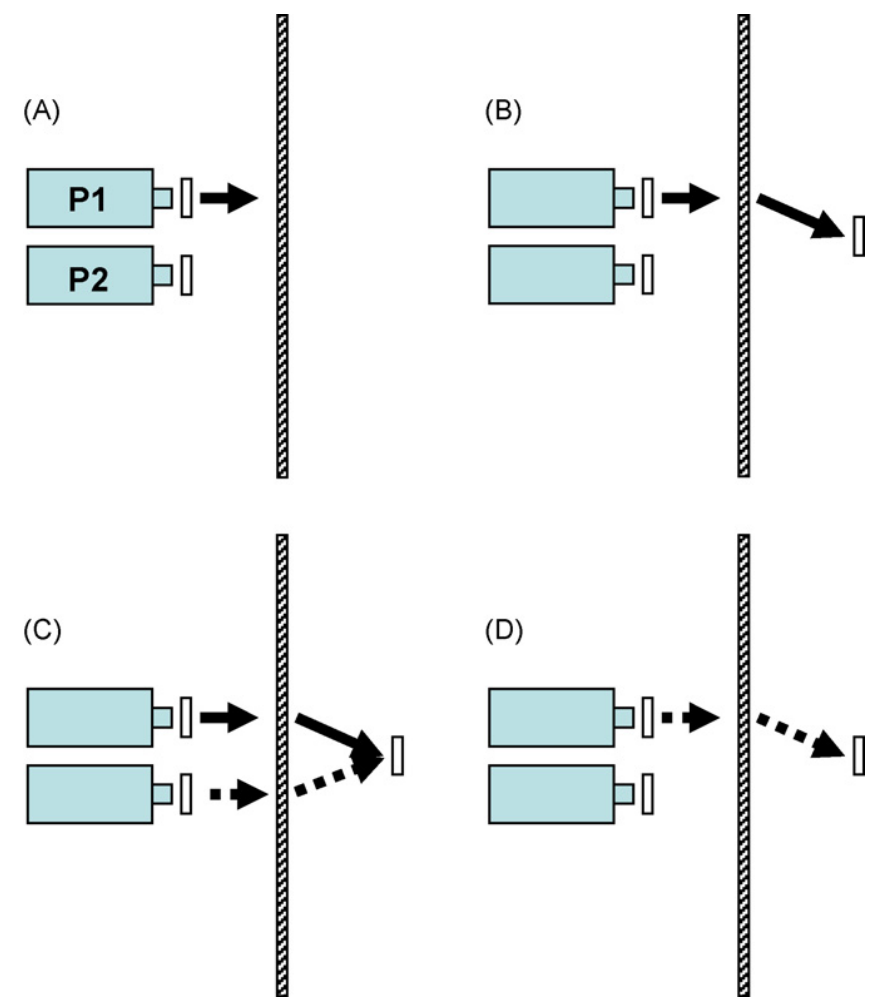

Fig. 2. A schematic representation of the projection and polarization arrangements for the four experimental conditions. Solid lines denote light to which the second polarizer (when present) is open. Dashed lines denote light polarized orthogonally to the second polarizer. P1 is the first projector and P2 the second projector. 
ing the subject's eye caused by the polarization process alone (Fig. 2B). A second projector was then added to this configuration projecting mean luminance at an orthogonal polarization whilst a set of measurements was repeated for the first projector (Fig. 2C). This was done to assess the amount of "cross-talk" between the orthogonally polarized projected images. Finally measurements were made for one projector through a closed polarizer to assess the amount of "leakage" that could occur (Fig. 2D). Here we define cross-talk as the amount of luminance from the image projected to one eye obtained in the image projected to the other eye. Cross-talk is a direct result of light leakage through an optical system that is designed to block that light. Both cross-talk and leakage were examined as a function of luminance linearity, a fundamental component in studies investigating visual perception.

\section{Results}

As can be seen in Fig. 3A and B, the presence of the second open polarizer reduced the slope of the physical luminance output as a function of increasing gray level. The slope decreased by a factor of 3.5 (3.2 vs. 0.9, excluding the final two measure- ments which plateaued for both sets of measurements). On its relative scale, this reduced output was still linear. Importantly there was no effect of adding a second, orthogonally polarized, projector to the single projector measurement (Fig. 3A and B). Finally if the polarizer was closed to a single projector there was little change in luminance with increasing gray level (Fig. 3A) although with a fine enough scale, it is apparent that the increase in luminance that did occur followed the same pattern as seen in the other conditions (Fig. 3C). This increase in luminance equates to approximately a quarter of a percent increment on any image presented to that eye, rendering the leakage undetectable under normal viewing conditions.

\section{Discussion}

The stimulus display system described in the current report allows for the accurate projection of dichoptic stimuli within an MR environment without the need to sacrifice any stimulus properties such as color, resolution or size. Additionally, the system described and verified above can be constructed with minimal financial cost when compared to other commercially available systems boasting high stimulus control and accuracy (e.g., fMRI
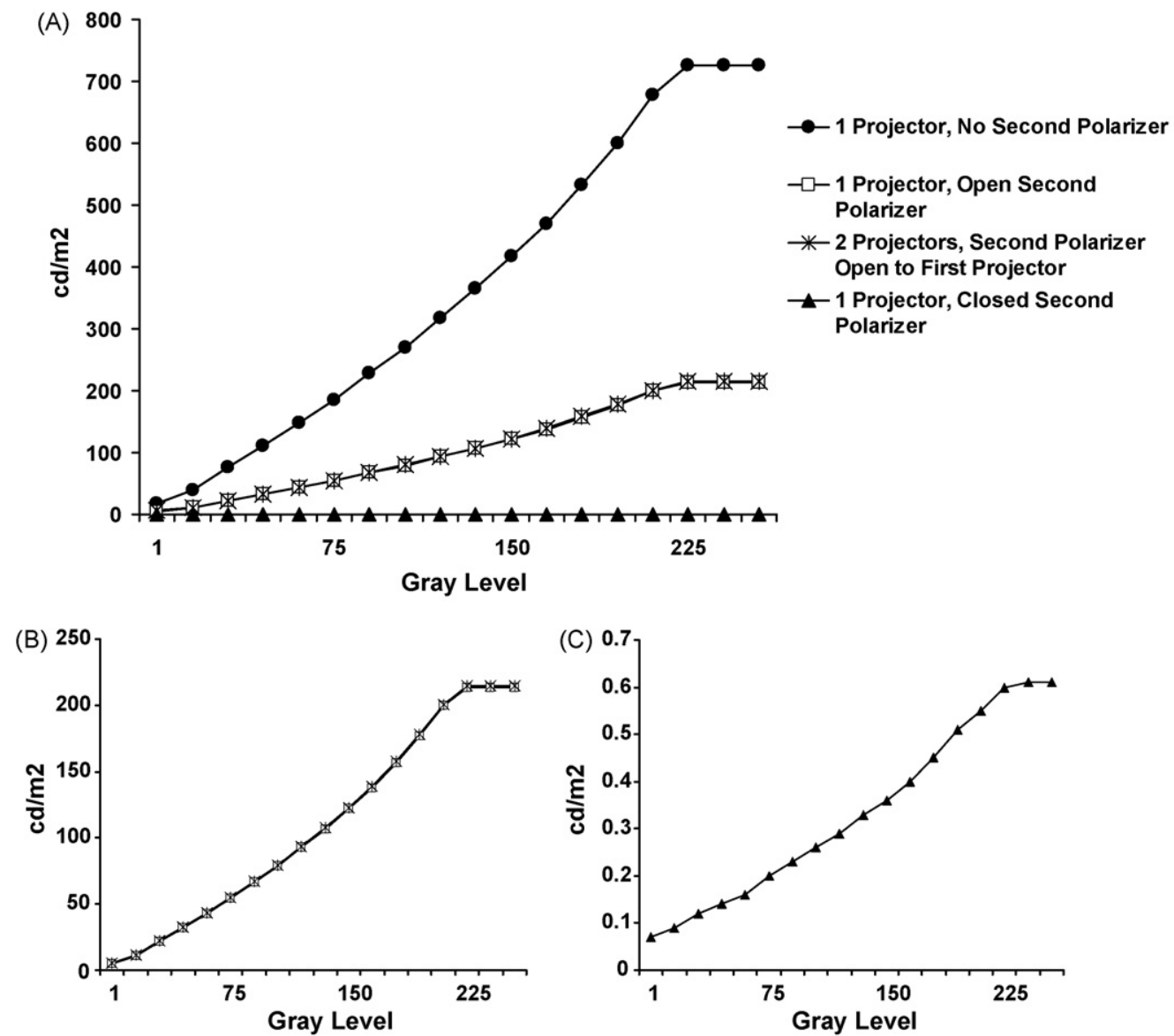

Fig. 3. Measures of the luminance reaching the eye as a function of gray level under four different projector and polarization arrangements. Results are shown for for 1 projector alone with no second polarizer (filled circles), 1 projector with an open second polarizer (open squares), the same configuration with the addition of a second projector projecting mean luminance with a polarization orthogonal to the second polarizer (asterisks) and finally 1 projector with a closed second polarizer (filled triangles). Panel A shows all four measurements on the same ordinate scale. To show each dataset more clearly the two conditions of interest showing the effect of adding a second projector are plotted alone in panel B. Finally the closed polarization condition is plotted alone in panel $\mathrm{C}$ using a significantly finer ordinate scale. 
compatible binocular goggles), and offers a more comfortable experience for the subjects. The components used in the system described here cost approximately $\$ 7000$ at the time of writing. Furthermore, projection systems are constantly being improved in terms of resolution and refresh rate in order to meet consumer demands for High Definition television. The current projection apparatus would easily allow for projector upgrades as technology develops. Such upgrades are substantially more difficult with goggle systems. The projection system uses linear polarizers to separate the images from two separate projectors projecting onto the same rear-projection screen. The polarizers are mounted both in front of each projector and in front of the subject's eyes to allow for dichoptic stimulation. The linearity verification experiment described above demonstrated that the system has minimal "cross-talk" between the images projected to each eye since the image from one projector is virtually extinguished by an orthogonally oriented polarizer mounted in front of the eye. This system may be of interest to researchers planning fMRI studies of the effects of dichoptic masking, stereo images, binocular rivalry and inter-ocular suppression on neural activity.

There are some factors that must be considered before this system is deployed within an imaging facility. Firstly, it should be noted that this system should optimally be situated inside the scanner room, as the technique of projecting through an aperture in the wall employed in some imaging centers would prevent the use of two projectors, unless the aperture was sufficiently large or additional optics (e.g., prisms) were used to merge the two images together prior to passing through the aperture. The second consideration regards eye tracking. Although theoretically infra-red illumination of the eye required by some MR compatible eye tracking systems should not be prohibited by the presence of polarizers over the eyes as the polarizers are not effective in the infra-red part of the spectrum, we would advise testing such an eye tracking system with a single polarizer held over the tracked eye before constructing the dichoptic projection system to verify that the eye tracking system can tolerate the presence of the polarizer. We have conducted this test using a laboratorybased infra-red eye tracking system (monocular infrared video Eyetracker and Eyetracker Toolbox, Cambridge Research Systems) and found that the polarizer did not prevent the infra-red camera from detecting the eye and that rotating the polarizer had no effect on the ability of the camera to detect the eye, suggesting that the polarizer was not effective in the infra-red range and would not impede eye tracking for this specific system.

Although other projector-based techniques for dichoptic presentation are available for the MR environment, these either require color changes to the images (anaglyph glasses) or are difficult to use in a range of scanner environments (binoculars + mirrors). fMRI compatible goggle systems are available which offer the ability to present stimuli dichoptically, however these systems can be prohibitorily expensive and often offer inferior image quality and control in comparison to projection systems. Given these considerations, the duel projection system described here allows researchers to conduct studies requiring dichoptic stimulus presentation in fMRI facilities not equipped with goggle devices or in situations where close control over stimulus attributes is required.

\section{Acknowledgements}

This research was supported by CIHR grant \#MOP 53346 to RFH. The authors would like to thank Mr. Walter Kucharski for manufacturing the components and Magda Michna, Elizabeth Wong and Kathy Mullen for helpful discussions.

\section{References}

Backus BT, Fleet DJ, Parker AJ, Heeger DJ. Human cortical activity correlates with stereoscopic depth perception. J Neurophysiol 2001;86:2054-68.

Baker DH, Meese TS, Summers RJ. Psychophysical evidence for two routes to suppression before binocular summation of signals in human vision. Neuroscience 2007;146:435-48.

Brouwer GJ, van Ee R, Schwarzbach J. Activation in visual cortex correlates with the awareness of stereoscopic depth. J Neurosci 2005;25:10403-13.

Chandrasekaran C, Canon V, Dahmen JC, Kourtzi Z, Welchman AE. Neural correlates of disparity-defined shape discrimination in the human brain. $\mathrm{J}$ Neurophysiol 2007;97:1553-65.

Fang F, He S. Cortical responses to invisible objects in the human dorsal and ventral pathways. Nat Neurosci 2005;8:1380-5.

Harrad RA, Hess RF. Binocular integration of contrast information in amblyopia. Vis Res 1992;32:2135-50.

Haynes JD, Rees G. Predicting the stream of consciousness from activity in human visual cortex. Curr Biol 2005;15:1301-7.

Haynes JD, Deichmann R, Rees G. Eye-specific effects of binocular rivalry in the human lateral geniculate nucleus. Nature 2005;438:496-9.

Kim S-C, Kim E-S. A novel configuration of LCD projectors for efficient orthogonal polarization of two projected views. Opt Commun 2006;266:55-66.

Levi DM, Harwerth RS, Manny RE. Suprathreshold spatial frequency detection and binocular interaction in strabismic and anisometropic amblyopia. Invest Ophthalmol Vis Sci 1979;18:714-25.

Li B, Peterson MR, Thompson JK, Duong T, Freeman RD. Cross-orientation suppression: monoptic and dichoptic mechanisms are different. J Neurophysiol 2005;94:1645-50.

Macknik SL, Martinez-Conde S. Dichoptic visual masking reveals that early binocular neurons exhibit weak interocular suppression: implications for binocular vision and visual awareness. J Cogn Neurosci 2004;16:1049-59.

Meese TS, Georgeson MA, Baker DH. Binocular contrast vision at and above threshold. J Vis 2006;6:1224-43 [electronic resource].

Meng M, Remus DA, Tong F. Filling-in of visual phantoms in the human brain. Nat Neurosci 2005;8:1248-54.

Moutoussis K, Keliris G, Kourtzi Z, Logothetis N. A binocular rivalry study of motion perception in the human brain. Vis Res 2005;45:2231-43.

Polonsky A, Blake R, Braun J, Heeger DJ. Neuronal activity in human primary visual cortex correlates with perception during binocular rivalry. Nat Neurosci 2000;3:1153-9.

Sengpiel F. Binocular rivalry: ambiguities resolved. Curr Biol 1997;7:R447-50.

Sengpiel F, Vorobyov V. Intracortical origins of interocular suppression in the visual cortex. J Neurosci 2005;25:6394-400.

Thompson B, Farivar F, Hess RF. Binocular Suppression in Amblyopia. Program No. 279.16. 2007 Neuroscience Meeting Planner. San Diego, CA: Society of Neuroscience; 2007 [Online].

Tong F, Nakayama K, Vaughan JT, Kanwisher N. Binocular rivalry and visual awareness in human extrastriate cortex. Neuron 1998;21:753-9.

Tong F, Meng M, Blake R. Neural bases of binocular rivalry. Trends Cogn Sci 2006;10:502-11.

Wunderlich K, Schneider KA, Kastner S. Neural correlates of binocular rivalry in the human lateral geniculate nucleus. Nat Neurosci 2005;8:1595-602. 